\title{
NTR Is the New SSTR? Perspective for Neurotensin Receptor 1 (NTR)-Directed Theranostics
}

\author{
Wolfgang P. Fendler ${ }^{1}$ and Richard P. Baum ${ }^{2}$ \\ ${ }^{1}$ Department of Molecular and Medical Pharmacology, David Geffen School of Medicine at UCLA, Los Angeles, California; and \\ ${ }^{2}$ Theranostics Center for Molecular Radiotherapy and Molecular Imaging, Zentralklinik, Bad Berka, Germany
}

See the associated article on page 936.

$\mathbf{E}$ small drugs with high affinity to target specific cell surface receptors of the cancer cell is highly effective and well tolerated. In this issue of The Journal of Nuclear Medicine, Schulz et al. report on neurotensin receptor 1 (NTR1)-directed RLT in a xenograft model of HT29 colon cancer (1). The affinity and specific uptake of the ${ }^{177} \mathrm{Lu}$-labeled antagonist 3BP-227 in NTR1-expressing tumors were established previously (2). In this preclinical model, Schulz et al. demonstrate high tumor-to-organ uptake ratios with effective and dose-dependent tumor growth inhibition (1). ${ }^{177} \mathrm{Lu}-$ 3BP-227 RLT exhibited a biodistribution and efficacy similar to those previously reported for ${ }^{177} \mathrm{Lu}$-DOTATATE $(3,4)$ or ${ }^{177} \mathrm{Lu}-$ PSMA (PSMA is prostate-specific membrane antigen) (5), drugs that already underwent successful translation. ${ }^{177}$ Lu-DOTATATE for RLT of metastatic neuroendocrine tumors proved highly efficacious, with a near $80 \%$ risk reduction for disease progression or death in a recently published prospective randomized international multicenter trial (6). ${ }^{177} \mathrm{Lu}$-PSMA led to high rates of biochemical and image-based responses in patients with metastatic prostate cancer (7). Both these treatments are exceptionally well tolerated, with a few serious, in most cases reversible, hematologic adverse events, and present milestones in the care of patients with metastatic neuroendocrine neoplasms or prostate cancer. Although the future application of NTR1-directed RLT is not yet clearly defined, there is a variety of indications to which it may be applied. NTR1 expression is seen in a range of cancer types, including colon carcinoma; pancreatic ductal adenocarcinoma; breast, prostate, and lung cancer; and head and neck squamous cell carcinoma (8). However, the level of NTR1 expression varies considerably.

For clinical translation, it is now critical to define a key indication based on clinical need, tumor biology, and target

\footnotetext{
Received Feb. 19, 2017; revision accepted Feb. 21, 2017.

For correspondence or reprints contact: Wolfgang Peter Fendler, University of California at Los Angeles, Ahmanson Translational Imaging Division, 10833 Le Conte Ave., 200 Medical Plaza, Ste. B114-61, Los Angeles, CA 900957370.

E-mail: wfendler@mednet.ucla.edu

Published online Mar. 9, 2017.

COPYRIGHT ( 2017 by the Society of Nuclear Medicine and Molecular Imaging. DOI: 10.2967/jnumed.117.191528
}

expression to subsequently prove efficacy of ${ }^{177} \mathrm{Lu}-3 \mathrm{BP}-227$ RLT in clinical trials. Stratification by PET/CT, as implemented for theranostic ${ }^{68} \mathrm{Ga} /{ }^{177} \mathrm{Lu}$ ligand pairs, is successfully applied within somatostatin receptor- and PSMA-directed RLT. The quest for a key application should similarly be guided by NTR1-directed PET/CT as gatekeeper for NTR1-directed therapy.

Pancreatic ductal adenocarcinoma (PDAC) was discussed as a potential key application based on high receptor density in about $75 \%$ of differentiated tumors $(9,10)$ and immense clinical need. Preliminary experience in a patient with metastatic PDAC indicated safety and effective tumor shrinkage after ${ }^{177} \mathrm{Lu}-3 \mathrm{BP}-227$ RLT (11). Therefore, metastatic PDAC might be the leading contender for NTR1-directed RLT, in particular because target expression was demonstrated in distant metastases (9). However, the efficacy of external radiation therapy for PDAC remains controversial $(12,13)$, indicating potential resistance mechanisms. Also NTR1 is absent in about one third of primary or metastatic lesions (9), underlining the need for individual patient stratification by NTR1directed PET/CT.

To define key indications for ${ }^{177} \mathrm{Lu}-3 \mathrm{BP}-227$ RLT, more data need to be acquired for PDAC and other cancer types. Target expression, tumor biology, and clinical need have to be considered to identify the most promising application to ultimately warrant successful clinical translation.

\section{DISCLOSURE}

No potential conflict of interest relevant to this article was reported.

\section{REFERENCES}

1. Schulz J, Rohracker M, Stiebler M, et al. Proof of therapeutic efficacy of a novel ${ }^{177} \mathrm{Lu}$-labeled neurotensin receptor 1 antagonist in a colon carcinoma xenograft model. J Nucl Med. 2017;58:936-941.

2. Schulz J, Rohracker M, Stiebler M, et al. Comparative evaluation of the biodistribution profiles of a series of nonpeptidic neurotensin receptor-1 antagonists reveals a promising candidate for theranostic applications. $J$ Nucl Med. 2016;57:1120-1123.

3. Dalm SU, Nonnekens J, Doeswijk GN, et al. Comparison of the therapeutic response to treatment with a ${ }^{177} \mathrm{Lu}$-labeled somatostatin receptor agonist and antagonist in preclinical models. J Nucl Med. 2016;57:260-265.

4. Lewis JS, Wang M, Laforest R, et al. Toxicity and dosimetry of ${ }^{177}$ Lu-DOTAY3-octreotate in a rat model. Int J Cancer. 2001;94:873-877.

5. Chatalic KL, Heskamp S, Konijnenberg M, et al. Towards personalized treatment of prostate cancer: PSMA I\&T, a promising prostate-specific membrane antigentargeted theranostic agent. Theranostics. 2016;6:849-861. 
6. Strosberg J, El-Haddad G, Wolin E, et al. Phase 3 trial of ${ }^{177}$ Lu-dotatate for midgut neuroendocrine tumors. N Engl J Med. 2017;376:125-135.

7. Rahbar K, Ahmadzadehfar H, Kratochwil C, et al. German multicenter study investigating ${ }^{177} \mathrm{Lu}$-PSMA-617 radioligand therapy in advanced prostate cancer patients. J Nucl Med. 2017;58:85-90.

8. Morgat C, Mishra AK, Varshney R, et al. Targeting neuropeptide receptors for cancer imaging and therapy: perspectives with bombesin, neurotensin, and neuropeptide-Y receptors. J Nucl Med. 2014;55:16501657.

9. Körner M, Waser B, Strobel O, Buchler M, Reubi JC. Neurotensin receptors in pancreatic ductal carcinomas. EJNMMI Res. 2015;5:17.
10. Reubi JC, Waser B, Friess H, Buchler M, Laissue J. Neurotensin receptors: a new marker for human ductal pancreatic adenocarcinoma. Gut. 1998;42:546-550.

11. Kulkarni H, Schuchardt C, Wiessalla S, et al. Radiopeptide therapy using Lu-177 3BP-227 in a patient with pancreatic adenocarcinoma [abstract]. J Nucl Med. 2015;56(suppl 3):1235.

12. Huguet F, Girard N, Guerche CS, Hennequin C, Mornex F, Azria D. Chemoradiotherapy in the management of locally advanced pancreatic carcinoma: a qualitative systematic review. J Clin Oncol. 2009;27:2269-2277.

13. Neoptolemos JP, Stocken DD, Friess H, et al. A randomized trial of chemoradiotherapy and chemotherapy after resection of pancreatic cancer. $N$ Engl J Med. 2004;350:1200-1210. 